\title{
Levels, Time and Fitness in Evolutionary Transitions in Individuality
}

\author{
Pierrick Bourrat ${ }^{\S}$
}

\begin{abstract}
In this paper, I identify two major problems with the model of evolutionary transitions in individuality (ETIs) developed by Michod and colleagues, and extended by Okasha, commonly referred to as the "export-of-fitness view". First, it applies the concepts of viability and fertility inconsistently across levels of selection. This leads Michod to claim that once an ETI is complete, lower-level entities composing higher-level individuals have nil fitness. I argue that this claim is mistaken, propose a correct way to translate the concepts of viability and fertility from one level to the other and show that once an ETI is complete, neither viability nor fertility of the lower level entities is nil. Second, the exportof-fitness view does not sufficiently take the parameter of time into account when estimating fitness across levels of selection. As a result fitness is measured over different periods of time at each level. This ultimately means that fitness is measured in different environmental conditions at each level and misleads Okasha into making the claim that the two levels are ontologically distinct levels of selection. I show that once fitness is measured over the same period of time across levels, the claim about two levels of selection can only be an epistemic one.
\end{abstract}

\section{KEYWORDS}

levels of selection $\bullet$ MLS1 $\bullet$ MLS2 $\bullet$ major transitions $\bullet$ evolutionary transitions in individuality

\section{Introduction}

Yes, fitness is the central concept of evolutionary biology, but it is an elusive concept. Almost everyone who looks at it seriously comes out in a different place. (Leigh Van Valen 1989,2-3)

ETIs are events in the course of evolution that lead to the formation of new higher level individuals due to the cooperation of two or more individuals at a lower level of organization (Michod 2011). One example of an ETI is the transition from uni- to multicellular organisms. A number of other ETIs have been proposed, among them the transitions from prokaryote to eukaryote cells and from multicellular organisms to integrated colonies (such as colonies of ants or honeybees). The most accomplished model of ETIs is certainly the one proposed by Michod and colleagues. In a number of articles and books (Michod 1999, 2005; Michod, Nedelcu, \& Roze 2003; Michod, Viossat, Solari, Hurand, \& Nedelcu 2006) they detail the necessary conditions for an ETI to occur. Okasha $(2006,2011)$ has recently set Michod and colleagues' work in the framework of multilevel selection 1 (MLS1)/ multilevel selection 2 (MLS2).

Okasha's and Michod's models of ETIs are committed to a concept of fitness which is measured by the ability of a given entity to survive (viability) and reproduce given that it has survived (fertility) in its

$\S$ Department of Philosophy,, School of Philosophical and Historical Inquiry, The University of Sydney, Faculty of Arts, Main Quadrangle A14 Sydney, NSW 2006, Australia

E-mail: pbourrat@gmail.com

Received 2 June 2014; Revised 3 September 2014; Accepted 11 February 2015 
environment. According to this definition, the higher the product of viability and fertility of an entity is, the higher its fitness is (see Sober 2001 for more on the way of conceptualizing fitness). Although much debate exists in the literature about the status of fitness (Ariew \& Lewontin 2004; Godfrey-Smith 2009; Krimbas 2004; Sober 2001) and whether 'fitness' consistently refers to the same concept (Michod 1999, 222), I will accept this definition as common ground for the development of my arguments. The main aim of this article is to argue against Michod's and Okasha's view of ETIs and more precisely against two specific claims defended by both authors. After having briefly reviewed Michod and colleagues' as well as Okasha's models of ETIs with respect to fitness, I will present these two claims: (1) during the last stage of an ETI, once a division of labor is in place, the fitness of the entities constituting the newly emerged individual becomes nil; (2) there are two fundamentally distinct processes of selection, namely multilevel selection 1 (MSL1) and multilevel selection 2 (MLS2), occurring alternately at the different stages of an ETI.

Claim (1) has recently been briefly criticized by Godfrey-Smith $(2011,77-78)$ for its metaphorical nature. In Section 3, I propose a more thorough examination of this claim and show first that Michod and colleagues' model relies on an assumption regarding fitness of the higher-level entity (hereafter the "collective") that is inconsistent with the claim made about the fitnesses of the lower level entities (hereafter the "particles"). ${ }^{1}$ I claim that an ambiguity surrounding the concept of viability (fertility) of the particle/ viability (fertility) of the collective exists in Michod's and Okasha's writings and creates the illusion that the fitness of the particle can be nil while the fitness of the collective is high. I also propose that even in cases where the fertility of the particles is literally nil, the concept of reproduction used by Michod and Okasha relies too much, although not explicitly, on material overlap such as is proposed by Griesemer (2000). I argue that this material overlap is unnecessary for Darwinian evolution to occur. Expanding on Godfrey-Smith (2009) and his concept of formal reproduction I show that somatic cells of multicellular organisms formally reproduce and consequently that their fitness is not nil.

In Section 4, I turn to claim (2) and demonstrate that if fitness is assessed over the same period of time at the collective level and at the level of its particles, then there most likely is commensurability between selection at the particle and collective level during an ETI. For that reason, they should not be claimed to represent two ontologically distinct processes of selection. I propose rather that they represent two ways of describing one and the same process from the perspective of two spatial and temporal scales. I do not deny the epistemological virtues of describing ETIs within the MLS1/MLS2 framework, but ultimately conclude that, so far, there is no clear evidence to support the claims given by Okasha about ontology. ${ }^{2}$

\section{Michod and Okasha on Evolutionary Transitions in Individuality}

Michod (2005) proposes the following model of ETIs. For new individuals at the collective level to emerge from the particle level (e.g. for multicellular organisms to emerge from unicellular organisms) two things must happen. First, conflicts between members of the collective need to be resolved. Two ways this can come about are the presence of policing mechanisms in the collective and the presence of developmental bottlenecks during life cycles. These two mechanisms lead to genetic homogeneity that reduces genetic conflict between the cells of an adult multicellular organism. However, even if genetic homogeneity between the different members of the same group is reached, this will not necessarily lead to the emergence of a higher level individual. For an ETI to take place, Michod (2005) proposes that there must be a division of labor between germ and soma (or its equivalent in ETIs other than from uni- to multicellular organisms), since without this division of labor, he argues, the collective fitness will remain strictly proportional to the average particle fitness. As such, the collective will not be an individual with its own fitness (Michod 2005, 569); its fitness will merely be a cross-level by-product ${ }^{3}$ of its particles' fitnesses. Thus, for Michod, the concept of individuality involves irreducibility of fitness at that level.

\subsection{Claim 1}

Michod defines the fitness of an entity (whether particle or collective) as the product of its viability and fertility, as it is often done in life-history models. When a cell is not specialized, it invests its resources in both 
the viability and fertility components of fitness. As a result its fitness is positive. However, when a cell invests everything in viability or everything in fertility, its fitness becomes nil because its product of viability and fertility is zero. Michod (2005, 2011) and Okasha (2009) both generalize this argument to other ETIs and propose that:

(1) If a particle invests everything in the somatic (or germ) function (or its equivalent) of the future collective individual, that particle's fitness will be nil since although its viability (or fertility) will be positive, its fertility (or viability), and consequently the product of viability and fertility, will be nil.

Conversely, they propose that when the two types of particles combine their investment in both components of fitness (one investing everything in the soma and the other everything in the germ function) a new collective individual emerges with its own fitness. This reasoning leads Michod to claim that during an ETI, there is a transfer or export of fitness from the particle to the collective level.

\subsection{Claim 2}

Okasha (2006) and Michod (2005, 2011), mostly relying on Okasha's analysis, both link this work to the two concepts of multilevel selection distinguished by Damuth and Heisler (1988), namely MLS1 and MLS2. In the MLS1 framework, the focal unit of selection is the particle. For that reason fitness is expressed as a number of particles produced. One collective has a higher fitness 1 than another if ceteris paribus it produces more particles. In the MLS2 framework, the focal units of selection are both the particle and the collective. Fitnesses of the collective and of the particle are measured in different units. The fitness 2 of a collective is expressed as the number of new collectives it produces independently of the number of particles composing each collective, while the fitness of a particle is simply expressed as the number of particles it produces. It should be noted that this way of presenting the MLS1/MLS2 framework is a simplification of Damuth and Heisler's (1988) original framework. Under their framework, not only do fitnesses refer to different variables, but in a MLS1 process the character under selection is a particle character, while in a MLS2 case the character under selection is a collective character. Following this latter approach, it might be justified to claim that MLS2 is ontologically different from MLS1. My targets in the present article are Michod's and Okasha's interpretations of the MLS1/MLS2 framework.

Okasha (2006, 237-238) proposes that an ETI can be schematically decomposed into three temporal stages for which MLS1 and MLS2 are alternately more relevant to describe the selection process(es) occurring at that stage. According to Okasha, in the first stage of an ETI the particles of the future collective start to aggregate and cooperate. The fitness of this newly formed collective is merely the average of the particles' fitnesses, hence MLS1 is the relevant type of selection occurring. During the second stage, the fitness of the collective is not defined in terms of the particles any more, but is proportional to the average of the particles' fitnesses. At that stage, although the MLS2 framework can be applied, so can the MLS1 framework. There is a "grey area between MLS1 and MSL2", in Okasha's words (2006, 237). However, the collective lacks individuality, since its fitness is a cross-level byproduct of the particles' fitnesses. During the third stage, when the transition is complete, the fitness of the collective cannot be expressed as the average fitness of the particles any more. The collective is now an individual on its own and its fitness is not proportional to the fitness of the particles; both fitnesses are now incommensurable. ${ }^{4}$ This leads Okasha to propose that:

(2) MLS1 and MLS2 are two ontologically distinct causal processes of selection, as opposed to two conventional ways of expressing selection (2006, 59; 2011, 243). During an ETI, they represent a transition in processes of selection. Not only are MLS1 and MSL2 alternately more relevant at the different stages of an ETI, they are alternately the only way to accurately describe those processes of selection. 


\section{Particles with Nil Fitness?}

In the previous section I presented (1), the claim that for an ETI from uni- to multicellular organisms (or more generally from particle to collective) to be successful, cells must renounce their individuality by specializing either in the soma or the germ function of this higher level individual. As a consequence of this specialization, Michod claims, their fitness taken separately is nil. Godfrey-Smith (2011, 77-78) briefly comments that this way of speaking about fitness is metaphorical. Indeed, he remarks that even in a paradigmatic case of multicellular organisms germ cells do not have a viability nil and many somatic cells divide, hence their fertility is not nil either.

In this section, I start by making Godfrey-Smith's remark more precise and show that one assumption in Michod and colleagues' model, namely that the viability and fertility of the multicellular organism are fully and only dependent respectively on the viability and fertility of the cells it is composed of, is clearly misleading. At that stage, it will become clear that Michod and Okasha confuse viability and fertility of the cells with viability and fertility of the multicellular organism. Intuitively, it might make sense to consider the fitness of somatic cells as nil. However, in the second part of this section I show that even if the fertility of somatic cells can be conceptualized as nil, this comes from the fact that Michod and Okasha have a classical understanding of the concept of reproduction which involves material overlap (for more on reproduction with material overlap see Griesemer 2000, 2014), which in Godfrey-Smith's view unnecessarily constrains the idea of evolution by natural selection. Following Godfrey-Smith, I propose a broader concept of reproduction, namely formal reproduction (Godfrey-Smith 2009, 79-81), to be used when describing foundational Darwinian processes such as ETIs. I show that if the concept of formal reproduction is used to measure the fitness of somatic cells in multicellular organism, they do not have a fitness nil because they formally reproduce.

\subsection{Viability and fertility at different levels}

Once an ETI from uni- to multicellular organisms with germ soma separation is completed, both types of cell in the organism cannot exist in the long term without each other. Without germ cells, the organism would sooner or later die without being able to reproduce. Without somatic cells, the organism could not survive long enough to reproduce. From a multicellular-organism perspective, it is therefore correct to state that without taking both the germ and somatic lines into account to measure fitness at the multicellular level, the fitness of the organism after one generation would be nil. But that claim is a different one from the one made by Michod and colleagues, which is that the fitness of the cells becomes nil once the ETI is completed (Michod 2005). There is obviously a sense in which the fitness of such a cell could be assumed to be nil. This would be when the fitness of a cell refers to the fitness that this cell would have had in the absence of a cellgroup environment. But this definition of fitness cannot play any causal role in ETIs. This is because a situation in which cells have no cell-group environment does not correspond to an actual situation encountered by the cells. In fact, the cells that Michod claims have a nil fitness are always (or at least most of the time) surrounded by a cell-group environment. It thus seems incorrect to claim that their fitness is nil and would be more accurate to claim that their fitness would have been nil. Making this sort of counterfactual claims can be useful in explanations but cannot be invoked for claims about ontology. Similarly, claiming that you would have had a nil fitness if you had not received milk from your mother does not mean that your fitness is nil, although it could be a useful claim to make for some particular explanation. Thus, although it might be justified to claim that a new individual emerges when one can identify a germ-soma specialization, equating viability and fertility of the particle-level entities (the cells) with viability and fertility of the collective-level entity (the multicellular organism) seems misguided.

It is clear that a causal relation between viability and fertility at the two levels exists, but viability and fertility of a cell and of a multicellular organism clearly refer to different processes in most instances of multicellular organisms. To clarify this point, let us take a hypothetical multicellular organism such as the one used or modeled by Michod and co-workers (of the genus Volvox) in different articles (e.g., Michod, et al. 2003; Michod \& Roze 1999; Michod, et al. 2006). Let us imagine that this multicellular organism starts to develop through the multiplication of two lines of cells: one that will perform the somatic function of the 
multicellular organism (for example keep the multicellular organism afloat), and one that will perform the germ function of the multicellular organism and will, once the organism has reached adulthood, give birth to new multicellular organisms.

In this hypothetical case, the terms 'viability' and 'fertility' at the collective level do not respectively equate to the terms 'viability' and 'fertility' at the particle level. In fact, during the phase of development of the multicellular organism - that is, when only the somatic or viability function of the organism is performed - the somatic line has offspring, and to do so the somatic cells need both to survive and reproduce. Similarly, during the phase of reproduction of the multicellular organism the germ cells both survive and reproduce. If this reasoning is correct, it means that the only difference between the developmental and reproductive phases of the multicellular organism from the point of view of cells is the distribution of their offspring cells, either in the multicellular organism for somatic cells or its offspring for the germ cells. Hence, not distinguishing the level at which the concepts of viability or survival and reproduction or fertility are applied can lead to the odd claim that the fitnesses of the cells of a multicellular organism are nil when those cells do survive and reproduce. Another way to make the same point is to say that the viability and fertility of the multicellular organism result from the effort of the cells in those variables. Yet, if the invested effort of a particular line of cells in the viability (fertility) of the multicellular organism is nil, it does not follow that the viability (fertility) of the cell is nil.

This confusion is most vivid where Okasha summarizes Michod's model of ETI from unicellular to multicellular organisms. Okasha writes:

[Let us] consider the extreme case where there is complete germ-soma specialisation - a cell either focuses entirely on viability (so [fertility of the $i^{\text {th }}$ cell:] $b_{i}=0$ ), or entirely on fecundity (so [viability of $i^{\text {th }}$ cell:] $\left.v_{i}=0\right)$. Clearly, this means that each cell has an individual fitness $\left(v_{i} b_{i}\right)$ of zero, so average cell fitness $C$ is zero too. Group fitness $G$, however, defined in Michod's way, may be very high - essentially, because some cells' null investment in $\mathrm{v}$ (or $b$ ) may be offset by other cells' high investment in $v$ (or $b$ ). Had group fitness been defined as average cell fitness, this would not be possible (Okasha 2009, 567). ${ }^{5}$

What seems to be a confusion in this quote is that a cell focuses entirely on the viability or fertility of the group, and not on the viability and fertility of the cell as Okasha suggests when he writes $b_{i}=0$ and $v_{i}=0$. In the case described by Okasha, only the effort in and $V$ and $B$ (respectively viability and fertility of the group) are nil.

In their models, Michod et al. assume that

...for simplicity, $[\ldots]$ the viability and fecundity of the group, $V$ and $B$, respectively, are simple additive functions of the cell properties given by $B=\sum_{i=1}^{N} b i_{\text {and }} V=\sum_{i=1}^{N} v i$ [where $b i$ and $v i$ are, respectively, the viability and fertility of the $i^{\text {th }}$ cell of the group] (Michod et al. 2006, 262).

They couple this assumption with the assumption that when an ETI is complete $v i=0$ and $b i=b m a x$ for the germ cells and $b i=0$ and $v i=\operatorname{vmax}$ for the somatic cells. This means that, at that point, $V$ and $B$ depend only on the viability of the somatic cells and the fertility of the germ cells, respectively. It may be biologically meaningful to assume that the viability of some multicellular organism depends solely on the viability of one particular type of cell that never reproduces, so that the effort in $V$ matches perfectly with the sum of the investment in $v$ of the cells. However, assuming that the fertility of the multicellular organism depends solely on the fertility of the cells - that is, that the effort in $B$ matches perfectly with the sum of the investment in $b$ of the cells that do not survive until reproduction - is biologically meaningless, since one necessary condition for having offspring is to be alive. In other words, following the assumption that the viability of the germ cells forming a multicellular organism is nil, the fitness of the whole organism should be nil because without existing, they could by definition not reproduce.

\subsection{Fitness and formal reproduction}

The previous argument only undermines Michod's export-of-fitness model with respect to the assumption that the fertility of the organism is only dependent on the fertility of the cells. But so far, the 
assumption that $V=\sum_{i=1}^{N} v i$ seems plausible, at least for some specific cases, ${ }^{6}$ such as when somatic cells never reproduce or when $b_{i}$ measures the fertility rate of cells across multicellular generations. In those cases, it can be tempting to assume, as do Michod and colleagues and Okasha, that the fitnesses of somatic cells are nil. In the remainder of this section I show that one should resist this temptation.

One way to show that the assumption that somatic cells have a nil fitness is misled involves defining 'reproduction' in a way that is necessary and sufficient for evolution by natural selection to occur. GodfreySmith $(2009,79-85)$ proposes that a concept of reproduction which necessitates material overlap, such as that proposed by Griesemer (2000), is not suitable for a foundational description of Darwinian processes. Griesemer (2000) argues that for evolution by natural selection to occur, similarity between parents and offspring is insufficient. Material overlap involves the offspring being made of the parts of their parents (2000, 74). Godfrey-Smith prefers the concept of formal reproduction, which only involves an unspecified relation of causality between the form of the 'parent' and of its 'offspring' and thus does not require material overlap between the two. This is the concept I follow here. The main argument Godfrey-Smith provides for preferring the notion of formal reproduction to the notion of reproduction with material overlap is that there are cases of in which there is no material overlap that are classically recognized as cases of evolution by natural selection. The clearest example used by Godfrey-Smith is of a retrovirus in which, due to reverse transcription, there is no parental material of the parent present in its offspring (GodfreySmith 2009, 80). Many more examples exist. ${ }^{7}$ In a recent article, Griesemer $(2014,36)$ asserts that he "accept[s Godfrey-Smith's] conceptual contrast of formal and material, but not his reasoning about which are the material cases". Griesemer shows that, depending on the scale or grain of description used and the choice of "central subjects" (a term borrowed from Hull (1975)), the case of the replicating retroviruses can be conceptualized in terms of material overlap. Although I accept that it might be possible to re-describe, in terms of material overlap, many cases that would under an intuitive or classical approach be recognized as cases of formal reproduction, this should not be a concern for me. It is sufficient that, at a given grain of description and after having chosen central subjects, material overlap will not be an important criterion to determine whether a population is evolving by natural selection. (For more on the notion of grain of description in evolutionary theory, see Bourrat 2014a, Chap 5).

In the case of reproduction of a multicellular organism, there seems to be no material overlap between the somatic cells of the parental organism and the offspring organisms. Thus, somatic cells are not the material parents of the offspring organisms. However, things are different if by reproduction we have in mind formal reproduction. In that case, one can argue that the somatic line of a multicellular organism does formally reproduce. To convince ourselves, let us imagine a case of a multicellular organism composed of somatic and germ cells. Let us further postulate that the fitness of the multicellular organism depends on both the number of somatic cells and number of germ cells it contains. If it is composed of an insufficient number of somatic cells its fitness plummets, for example because this low number does not allow the multicellular organism to remain afloat. Similarly, if it is composed of too many somatic cells (relatively to the total number of cells) its fitness also plummets because with a higher number of germ cells the organism could have produced more offspring organisms. The number of somatic cells in the multicellular organism is thus as important as the number of germ cell for the reproductive output of the organism.

For formal reproduction to happen, relatively to a particular grain of description, the only requirement is the presence of a causal link between the presence/absence of a somatic cell in the parental organism and the overall number of cells produced (number and quality of offspring). That is precisely what Michod et al.'s models test: "we considered how a single new cell could maximize its fitness contribution to the group. In the optimization model we test whether small deviations by two or more cells could increase the fitness of the group" (2006, 262). Indeed, in Michod et al.'s models one can establish the causal relation between the parental and offspring cells by manipulating the presence or absence of only one somatic cell in the parental organism and deduce that ceteris paribus one more somatic cell in the parental organism will lead to a higher number of offspring cells composing the offspring multicellular organisms (thus either a higher number or better quality of offspring organisms). Causation can thus be understood here under the manipulationist account of causation (Woodward 2003, 2013), although Michod and colleagues do not make such a connection. According to Woodward (2013) the cornerstone of the manipulative account of causation is 
that "if $C$ is genuinely a cause of $E$, then if I can manipulate $C$ in the right way, this should be a way of manipulating or changing $E$ ". In our case, manipulating the number of somatic cells in the right way has consequences for the fitness of the whole organism (see Figure 1 for a toy illustration).

In this scenario everything could be seen here as if the somatic cells were sending a message to the germ cells to reproduce more cells at the next generation. ${ }^{8}$ But does the fact that somatic cells are causally responsible for the production of somatic and germ cells not undermine my argument? In other words, is the fact that the manipulation of a number of somatic cells does not specifically alter the number of somatic cells in offspring organisms not a problem for my view? I claim that it is not. In fact, for evolution by natural selection to occur, perfect reproduction is unnecessary. The only requirement is that some information from parents and offspring is conserved. Moreover, in that respect, the concepts of formal reproduction and reproduction with material overlap are on a par. For example, germ cells in the parental organism also produce the somatic cells of the offspring organisms. The only important property that matters for somatic cells of a given organism with regard to natural selection is that their relative number, when compared to the overall population of organisms, increases over time. Whether this occurs through reproduction with material overlap or through formal reproduction does not matter. Elsewhere I develop the concept of formal reproduction using a set of agent-based models (see Bourrat 2014b). Another way to make this point is to notice that somatic and germ cells have a relatedness of 1 and thus, following standard kin selection theory (Hamilton 1964), the indirect fitness of somatic cells (as opposed to their direct fitness) is not nil. Yet, in kin selection theory the total personal fitness of an individual is measured as the sum of the direct and indirect components of fitness (see Gardner \& Foster 2008; West, Griffin, \& Gardner 2007). Thus having a nil direct fitness does not necessarily mean that the total personal fitness of a cell is nil. ${ }^{9}$

To sum up, I have thus far showed that an ETI does not lead the particles forming a collective to have a nil fitness, as it has been argued by Michod and Okasha. This becomes clear when one does not apply the concept of viability and fertility of the collective to the particles and when the classical concept of reproduction is substituted with the more general concept of formal reproduction. In this latter case, somatic cells do reproduce at each new multicellular generation of multicellular organism and consequently their fitness cannot be said to be nil.

\section{When Time Makes a Difference}

I turn now to claim (2). If claim (1) seems unjustified in light of the above arguments, the claim made by Okasha and Michod that during an ETI an ontologically new level of selection is created at the collective level, leading to two ontologically distinct levels of selections once the ETI is completed, remains untouched by my arguments so far.

According to Okasha, once an ETI is completed, the particle and collective fitnesses become incommensurable: one could not, even in principle, measure the fitness of the collective in terms of fitness of the particles. Where does such incommensurability come from? To this question there no clear answer is given, and it is hard to see how there could be one, even in principle. It is in fact hard to imagine that collectives could exhibit variations in fitness without their constitutive parts exhibiting a form of variation with consequences on their own fitness. Yet Okasha believes that such scenarios exist (Okasha 2006, 106) and that they materialize when MLS2 is the framework of choice. For the MLS2 framework, he claims, fits two causally distinct processes of natural selection happening in nature (Okasha 2006, 59; 2011, 243). Recall that in the MLS2 framework, the fitness of the collective can be defined as a quantity "that bears no necessary relation to average particle fitnesses alone" (2006, 136, my emphasis). Yet, in the same sentence Okasha surprisingly asserts that "it is impossible that the resulting evolutionary change could be expressed in terms of particle fitnesses alone" (Okasha 2006, 136, my emphasis). Beyond the fact that the claim does not follow from the premise (Okasha should have used "sometimes impossible" instead of "impossible"), I propose one important reason why we should doubt this claim in any case.

I will not argue here against the MLS2 framework itself, since it is true by definition. Rather, I will show that the claim that there is incommensurability between the particle and collective fitnesses in cases in which there is evolution by natural selection is unwarranted. The argument I provide is based purely on 


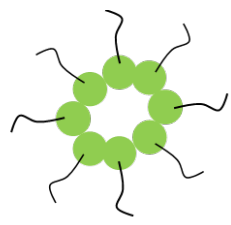

One multicellular organism containing 8 somatic cells

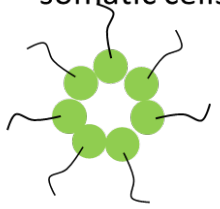

The same

multicellular organism

but containing only 7 somatic cells

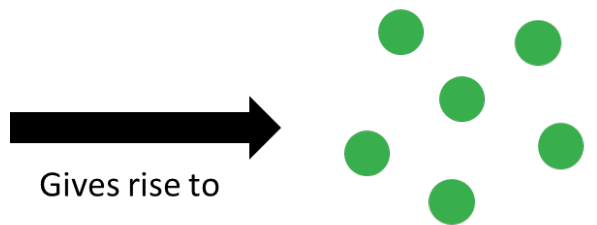

6 Multicellular offspring
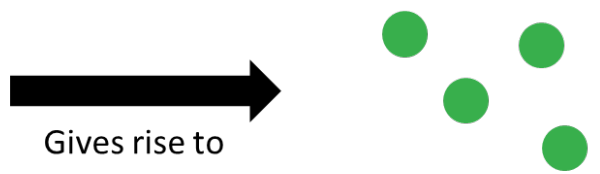

4 Multicellular offspring

Figure 1. Toy illustration of the formal causal relation between the presence/absence of one somatic cell in a multicellular organism of the genus Volvox and the number of multicellular offspring produced.

methodological grounds linked to time, fitness and levels of organization and I illustrate it with one of Okasha's own examples of MLS2.

In his chapter 7 Okasha (2006) deals with species selection, the paradigmatic case of MLS2 in the literature on the subject, and embraces Vrba's 'acid test' $(1989,155)$ to distinguish true species selection (and more generally MLS2) from mere by-products of selection at lower levels, as in MLS1. Vrba proposes that there is true species selection when the outcome of selection at the species level cannot be explained from the perspective of the organism. One stringent way (which represents the test) to know when this happens is to seek different directions of selection at the different levels of organization. For instance, species selection, if truly independent, could in principle counteract selection at the organism level. Vrba's test will however be inconclusive when both selection processes push in the same direction (see a discussion of this problem in Jablonski 2008). The most parsimonious attitude, that is the simplest one given the evidence, to adopt in such case will be to consider that selection only really occurs at the lower level (see Figure 2), unless one has some clear evidence of the contrary. Okasha $(2006,207)$ illustrates one example of true species selection satisfying Vrba's test with the evolution/maintenance of sexual reproduction. ${ }^{10} \mathrm{He}$ asserts, following a classical interpretation, that asexuality is advantageous at the organism level because of the two-fold cost of producing males (Maynard Smith 1978), but that sexuality is advantageous at the species level because it allows faster evolutionary responses to rapid changes in environmental conditions. According to this reasoning, sexual lineages would be selected via species selection as a process of natural selection distinct from selection at the organism level (which favors asexual organisms). ${ }^{11}$

One fundamental principle of experimental sciences, concomitant with the manipulationist account of causation, is that to establish causation, ideally one would change only one variable at a time while the others are kept unchanged or controlled in order to eliminate confounds. Controlled and randomised experiments rely on this idea since they are attempts to reach this goal (Fisher 1970; Shipley 2002). Thus to establish causation as opposed to a mere correlation, if one is interested in measuring the causal influence of $X$ (e.g., a drug) on a population $P$, the experimenter will need to control the effect of $X$ on $P$ with another population (let us call it $C$ for control) that was not administered $X$ but is as similar to $P$ as possible in all other respects. In such a case, if a difference is observed between the two populations, it will only be attributable to $X$ because no other variable is different. However, if $P$ and $C$ are not strictly identical in all respects other than $X$, then any observed difference could be attributable to $X$ or any of the other variables that differ between the two 


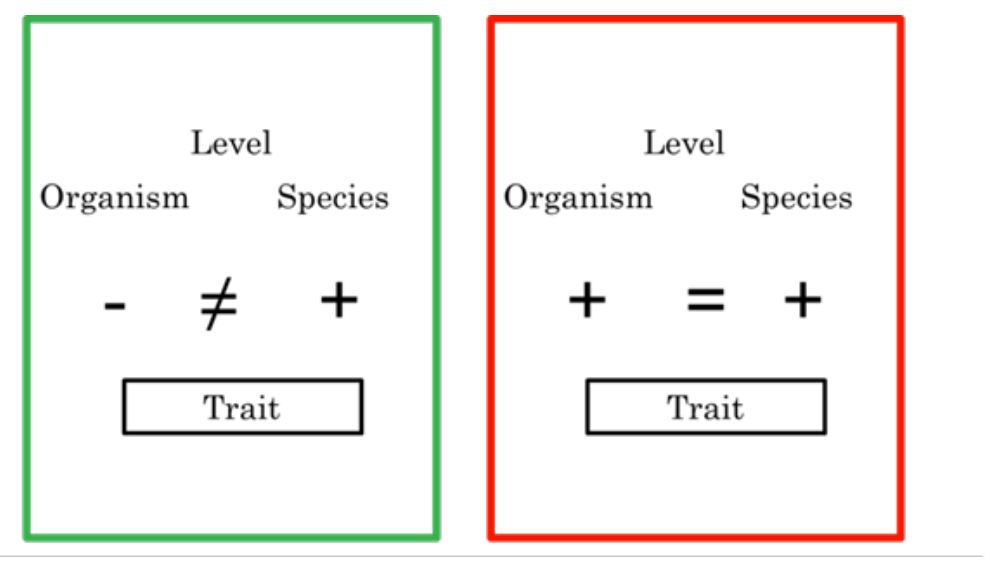

\section{Conclusive test Inconclusive test}

Figure 2: Vbra's acid test

populations and which could have the same effect as $X$. Such variables are classically called confounding variables. ${ }^{12}$

How is that relevant to our problem of species selection and Vrba's test? Vrba's test is not a scientific experiment per se, but it shares with them the necessity to be controlled. Unless all of the variables relevant to selection are strictly identical at both levels in the test, the detection of a different direction of selection at those levels could be attributed either to a different process of selection at each level or to any other variable with different values at each level and with some relevance to the direction of evolutionary change. Just like any scientific experiment, Vrba's test requires, ideally, that only one variable at a time is changed while all the others are kept unchanged.

We noted earlier that Okasha claims that the evolution/maintenance of sexual reproduction is a true case of species selection. He justifies this assertion using Vrba's test. Because, he argues, the test shows that selection pushes in two opposite directions (i.e. selection for sexuality at the species level and selection for asexuality at the organism level), a process of selection ontologically different from the process of selection at the organism level, must exist at the species level. But does Okasha's comparison eliminate all possible (or at any rate all known) confounding variables, which could render his conclusion spurious? In other words, is selection at the organism level assessed in the exact same way as it is at the species level? The answer to this question is that it is not; Okasha has overlooked the issue of confounding variables.

To detect this confounding variable, let us use Okasha's example once more and consider two types of organisms, one asexual and one sexual, under the same selection pressures. The so-called twofold cost of males occurs in sexual organisms, because only half of sexual organisms can reproduce (only the females) and they produce offspring of both sexes. On the contrary, asexual organisms are able to reproduce without partner and only produce female organisms. Hence if the two types of organisms are in competition, the asexual ones should quickly out-compete the sexual ones, because of the supplementary costs associated with sexual reproduction. A good example of this phenomenon within the same species is explained by Bouchard (2008, 2011). The Quaking aspen (Populus tremuloides) can reproduce both sexually and asexually, but Bouchard (2011) notes that the asexual clonal grove often beats the sexually reproducing individuals. In such cases, it is thus extremely tempting to claim that the fitness of an asexual organism is higher than the fitness of a sexual organism. In fact replacing "trait" by "sexual reproduction" or "asexual reproduction" in Figure 2 seems to yield two different levels of selection going in two opposite directions. But, if performed as such, the test is applied incorrectly. To apply it correctly, measures of fitness must be relativized over the same period of time at both levels of organization. This point has been made by Bouchard (2011) in the context of 
comparisons of fitness between different of organisms. I make a similar point in the context of comparisons of fitness between levels (see also Pocheville 2010, Chap. 2).

In an environment that is perfectly stable all the time, it is very likely that most of these proxies will be equally good. In such cases, having the reproductive output after one or after two generations, for instance, will make no difference in our estimation of whether natural selection occurs between two or more types in a population. However, if every two generations there are changes in the environment that affect the reproductive output of organisms differentially, the reproductive output after one generation will be misleading if one wants to know the evolutionary dynamics of a population over periods of time longer than two generations. Similarly, the reproductive output after two generations will be misleading if one wants to predict the evolutionary change for a period of time shorter than two generations.

Thus, there is no 'best' period of time over which one should measure fitness for there is no definite environmental conditions that are the 'true' environmental conditions of an organism. This has led Beatty \& Finsen (1989), Sober (2001), and more recently Abrams (2009) to propose a distinction between short-term and long-term fitness. Although they all consider that short-term and long-term reproductive outputs are fitness, it should be clear by now that my view is that they represent different proxies of fitness.

Proxies of fitness over long periods of time should be preferred if one is interested in evolutionary problems involving long term environmental changes, as is the case with the evolution and maintenance of sexual reproduction. This is because long term environmental changes and their consequences on selection pressures will be invisible to a proxy for fitness based on short term reproductive output. Increasing the period of time over which proxies of fitness are evaluated might represent a solution to problems identified by Ariew and Lewontin (2004). Yet, many evolutionary problems do not involve such changes and measuring fitness as the reproductive output over one generation is fine because the environment is stable enough. This is the case for instance if one wants to know what phenotype is optimal in a constant environment.

The confounding variable, or more precisely variables, in Okasha's comparison might now become more obvious to the reader. They are the environmental variables that changed with the measures of fitness made at the two levels. At the organism level, fitness is usually measured as the reproductive output after one organism's generation. At the species level, fitness is measured as the rate of extinction or speciation over much longer periods of time, sometimes many millions of years. Thoday (1953), for example, considers that fitness should be measured as the probability of leaving a descendent after a period of $10^{8}$ years. Speciation and extinction events are ultimately composed of the deaths, survivals and reproductions events of organisms over many generations, since the former events supervene on the latter. Thus, when Okasha applies Vrba's test over the maintenance/evolution of sex, he compares the fitness of organisms over one generation at the organism level with the fitness of organisms over a much higher number of generations. Performed as such, Vrba's test remains inconclusive. Indeed, the difference observed could be either due to two processes of selection pushing in opposite directions or to two measures of one and the same process of selection over different periods of time, pushing in one direction over the short term and in the other over the long term. In the rest of the article, I defend the latter possibility.

To see why, let us now perform Vrba's test while controlling the period of time over which fitness is measured (Table 1b). Controlling time could be done in two ways: (1) by measuring the two fitnesses (at both the species level and the organism level) over one organism generation and comparing them over this period of time; (2) by measuring the two fitnesses over the period of time that would normally be used to measure species' fitness, that is, a period long enough to detect events of speciation or extinction. Both alternatives seem to be doomed in practice, since we are neither able to measure the fitness of species over short periods of time, nor able to measure the fitness of organisms over periods of time longer than a few generations. But if we were able to do so, we would certainly find that ceteris paribus asexual organisms and asexual species have a higher short-term fitness as measured by (1) than sexual organisms and sexual species, but have a lower long-term fitness as measured by (2). The reason for this is not mysterious. Asexual organisms and asexual species on 


\begin{tabular}{|c|ccc|}
\hline Environment & Reproduction & organisms & species \\
\hline \multirow{2}{*}{ Not controlled for } & Asexual & + & - \\
\cline { 2 - 4 } & Sexual & - & + \\
\hline
\end{tabular}

Table 1a. Vrba's test comparing the direction of selection on the regime of reproduction (sexual or asexual) without controlling for the time over which fitness is measured in organisms and species

\begin{tabular}{|c|c|c|c|}
\hline Environment & Reproduction & organisms & species \\
\hline Constant - proxy: & Asexual & + & + \\
\hline short period of time & Sexual & - & - \\
\hline Changing - proxy: & Asexual & - & - \\
\hline long period of time & Sexual & + & + \\
\hline
\end{tabular}

Table 1b. Vrba's test comparing the direction of selection on the regime of reproduction (sexual or asexual) controlling for the period of time over which fitness is measured in organisms and species

average do better when the environmental conditions remain stable (as is usually the case over one generation) while sexual organisms and sexual species do better when new environmental conditions arise (which certainly occurs over several millions of years). In other words, both selection at the organism and the species level go in the same direction once the test is controlled for the period of time over which fitness is measured.

Table 1a represents Vrba's test performed on the regime of reproduction at the organism and species level when the confounding variable, time, a proxy of changes in the environmental conditions, is not taken into account. Table $1 \mathrm{~b}$ represents the same test performed when time is taken into account. We can see that contrary to what happens when time is not taken into account, we expect selection on the regime of reproduction to go in the same direction at the organism and the species level.

Thus, Okasha's claim that the evolution/maintenance of sexual reproduction occurs as a result of species selection is inexact. If we follow his reasoning, keeping the time over which fitness is assessed as a constant (meaning that the environment is the same at both levels), we predict no difference between a measure of selection made at the level of the organism and another one made at the level of the species. The simplest interpretation of difference in the direction of selection over time is that one and the same process of natural selection changes direction over time - not that two distinct processes are opposing each other (see also Bourrat 2015).

There is no logical barrier to extending this argument to all the other cases for which MLS2 has been the framework of choice. In each case, if fitness could be determined over the same period of time, or more precisely in the same environment, at each level, what seem to be ontologically different levels of selection could in principle be unified under one and the same process. Does this mean that the MLS2 framework should be abandoned and always replaced by the MLS1 framework? I claim that it should not, unless one has the full availability, at any point in time, of the selection pressures on the particles under consideration. I can only see simple multilevel models as satisfying these criteria. In practice, the complete list of selection pressures an entity is subjected to will most of the time be unknown, or they will be constantly changing (e.g. frequency dependent selection), making the particle fitnesses extremely complex to determine over long 
period of time. When both particle and collective fitnesses are available, and when the question at stake is about the collective, I propose that the MLS2 framework should be privileged. There are two further reasons for this choice. First, the hard task of measuring the fitnesses of all of the particles within a collective (with all the non-linear relations it implies) and over many particles' generations will often materialize at the collective level into a single and easily measurable parameter: the collective's reproductive output. Second, keeping fitness of the particles and fitness of the collective independent, as it is done in the MLS2 framework, can reveal different, relevant information about selection pressures (for they are measured over different periods of time).

In light of these general considerations on MLS2, what does the MLS1/MLS2 distinction become in the context of ETIs and especially during their last stage? Would it be, in principle, possible, at the last stage, to describe the fitness of a collective in terms of the fitnesses of its particles, contra Okasha? Following the reasoning I used in the case of the evolution/maintenance of sex, as in any case of MLS2, I see nothing that would prevent this, as long as a multicellular organism is nothing more than the cells it is composed of. ${ }^{13}$ During an ETI, if the fitness of the particles seems incommensurable with the fitness of the collective, it is most probably due to the fact that, during the last stage, those fitnesses are not measured over the same period of time anymore, and the interactions between particles become so complex that tracking back their fitness over longer periods of time than one or two generations appears in practice impossible. Yet, what becomes decoupled in the two levels is not fitness per se but generations or life cycles.

Because Michod's proxy for fitness depends on reproductive output after one generation, if "one generation" does not mean the same thing at the particle and the collective level, it is not surprising that collective and particle fitnesses seem decoupled from each other. This, in fact, is an artifact created by the measure. To see this, let us take again a take a toy example of hypothetical multicellular organisms of two types, A and B, that develop through cellular division and then reproduces at the multicellular level (see Figure 3).

Suppose that type A develops through a single totipotent cell (in red on Figure 3) into two totipotent cells, of which one will lead to two germ cells (in green on Figure 3) and the other to one germ cell and one somatic cell (in blue on Figure 3, which we suppose unable to reproduce). Finally, suppose that this hypothetical multicellular organism is able to produce three offspring. Suppose now that type B, instead of waiting until the second cellular generation to differentiate the totipotent cells as somatic and germ cells, does so at the first cellular generation. As a result, at the third cellular generation, the multicellular organism of type B is composed of two somatic cells and one germ cell; that is, one somatic cell more and two germ cell less than type A. But imagine now that the fact that type B has one more somatic cell than type A at the third cellular generation allows it to have three more cellular generations than type A before producing the multicellular offspring (see Figure 3). This could be explained, for instance, by supposing that somatic cells gather resources for the multicellular organism and that having more resources allows for more cellular reproduction and a higher number of multicellular offspring, with a priority given to the production of cellular offspring until a certain threshold of germ cell is reached and multicellular organisms are produced. In the case of type A in Figure 3, at the third cellular generation, all of the resources gathered go to the production of new multicellular offspring, while in the case of type $\mathrm{B}$, the resources gathered go to the production of new germ cells in the cellular generations 4 and 5 and from that point the ressource gathered go to the production of multicellular organisms. Because multicellular organisms of type $\mathrm{B}$ have one more germ cell and one more somatic cell than multicellular organisms of type A, they are able to produce one more multicellular offspring (as shown on Figure 3). I am assuming here that it takes the same absolute time to produce a new multicellular generation for both types and that multicellular organisms die immediately after reproduction.

As we can see in Figure 3, if we compare the measures of cell fitness of the two types with their measures of multicellular-organism fitness using, respectively, one cellular generation and one multicellularorganism generation, we find that type A has a cell fitness of 2 and a mutlicellular-organism fitness of 3, while type B has a cell fitness of $1.43^{14}$ and a multicellular-organism fitness of 4 . Thus A seems to be fitter at the cell level and B fitter at the multicellular level. We seem to be in a case where one process of selection pushes in one direction at the cell level and another in the other direction at the mutcellular-organism level (as in the case of species selection developped earlier). Yet, this artefact quickly disappears if we measure the 


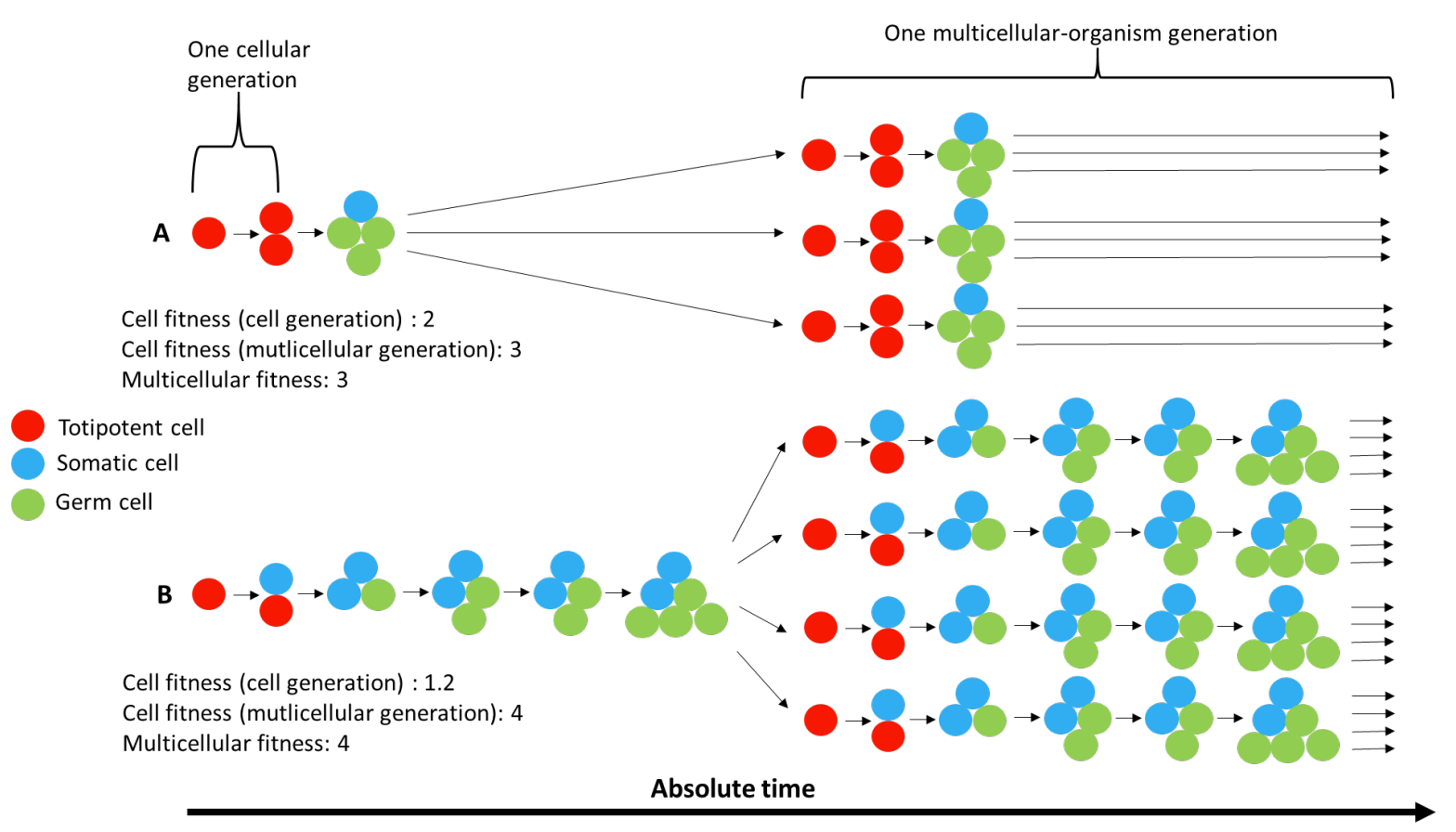

Figure 3. hypothetical case of multicellular organisms with different life cycles

fitness of the cells and multicellular organisms over the same period of time. Then we can see that both selection at the cell and at the mutlicellular-organism level go in the same direction. In the example proposed in Figure 3, type A has a cell fitness (measured over the muticellular-organism generation from birth to death) of 3 and type B of 4, which are the same values as those obtained at multicellular level.

Although the demonstrations provided here suggest that the MLS2 framework does not vindicate an ontologically distinct process of selection from MLS1, it does not render the notion of MLS2 useless. In fact, MLS2 is a very useful means to carve one single process of natural selection both in time and space, and becomes especially useful once an ETI is completed. This echoes a recent criticism made by Waters (2011) about Okasha's fundamentalism over the distinction between MLS1/MLS2 in which Waters claims that MLS1 and MSL2 frameworks are more conventional than fundamental. In a response Okasha $(2011,243)$ holds his ground, restating that they are fundamental. I have provided evidence here about their conventional status once measures of fitness are controlled appropriately.

\section{Conclusion}

In this article I have demonstrated three things. First, when doing a multilevel analysis of an evolutionary process, it is important to use comparable concepts at each level. The terms 'viability' and 'fertility' and consequently 'fitness' cannot be used interchangeably when referring to the cell (and more generally the particle) and the multicellular organism (and more generally the collective). Although Michod and colleagues' model formally distinguishes the two, it assumes a direct relation between viability and fertility of the cell and viability and fertility of the organism. I have shown that cell viability and fertility should not, to be biologically meaningful, be straightforwardly translated into viability and fertility of multicellular organisms, even in very simple cases (such as the ones proposed by Michod and colleagues).

Second, I have shown that the concept of formal reproduction can be useful to consider during ETIs and that it has the advantage of avoiding the odd claim that the somatic cells of a multicellular organism have a fitness nil.

Third, I have demonstrated that time is an extremely important parameter to take into account with respect to the concept of fitness and more precisely fitness measures. I argued for its relevance in ETIs and, 
more generally, in the levels of selection debate. I used the evolution/maintenance of sexual reproduction as an illustrative example to establish that, if different proxies of fitness reflecting different time scales are used at the organism and species levels, this will have the consequence of measuring selection pressures over two different sets of environmental conditions. This can lead one to confound the existence of one unique process of selection over two different periods of time with two ontologically distinct processes of selection, one for each level. I then applied the same reasoning to ETIs using a simple model and argued that they were not transitions in processes of selection, but rather events for which MLS1 and MLS2 were alternately more relevant.

The claim that distinction between collective selection and particle selection is conventional is not new (e.g. : Dugatkin \& Reeve 1994; Sterelny 1996) and Kerr \& Godfrey-Smith (2002) have formalized this equivalence. Yet, as Okasha $(2006,136)$ rightly points out, this formalism has been made solely in the context of MLS1. Taking time as an important variable in measures of fitness represents one important step towards a formalism in which any event of selection traditionally described under the MLS2 framework, such as the last stage of ETIs, could also be described under the MLS1 framework. A recent criticism, the exposition of which would go beyond the scope of this paper, calls into question the equivalence between particle and collective selection (conventionalism) in the MLS1 context (see Lloyd, Lewontin, \& Feldman 2008). This could undermine the claim I have made here about conventionalism between particle and collective selection. For my purposes here it suffices to note that whether this criticism holds is independent from the arguments provided in this paper, since the apparent incommensurability between fitnesses at different levels of organization at least partially results from an artefact of measuring fitness over different periods of time at different levels of organization. This is sufficient reason to take temporal scales seriously into account when making fitness comparisons.

\section{Literature cited}

Abrams, M. (2009). The Unity of Fitness. Philosophy of Science, 76(5), 750-761. doi: 10.1086/605788

Ariew, A., \& Lewontin, R. C. (2004). The confusions of fitness. The British Journal for the Philosophy of Science, 55(2), 347-363. doi: 10.1093/bjps/55.2.347

Beatty, J. H., \& Finsen, S. K. (1989). Rethinking the propensity interpretation: A peek inside Pandora's box. In M. Ruse (Ed.), What the Philosophy of Biology Is: Essays Dedicated to David Hull (pp. 17-30). Dordrecht: Kluwer Publishers. doi: 10.1007/978-94-009-1169-7 2

Bouchard, F. (2008). Causal processes, fitness, and the differential persistence of lineages. Philosophy of Science, 75(5), 560-570. doi: 10.1086/594507

Bouchard, F. (2011). Darwinism without populations: a more inclusive understanding of the "Survival of the Fittest". Studies in History and Philosophy of Science Part C: Studies in History and Philosophy of Biological and Biomedical Sciences, 42(1), 106-114. doi: 10.1016/j.shpsc.2010.11.002

Bourrat, P. (2014a). Reconceptualising evolution by natural selection. (PhD dissertation), University of Sydney, Sydney.

Bourrat, P. (2014b). From survivors to replicators: evolution by natural selection revisited. Biology \& Philosophy, 29(4), 517-538. doi:10.1007/s10539-013-9383-1

Bourrat, P. (2015). Levels of Selection Are Artefacts of Different Fitness Temporal Measures. Ratio, 28(1), 40-50. doi: $10.1111 /$ rati. 12053

Buss, L. W. (1983). Evolution, development, and the units of selection. Proceedings of the National Academy of Sciences, 80(5), 1387-1391. doi: 10.1073/pnas.80.5.1387

Buss, L. W. (1987). The Evolution of Individuality. Princeton, NJ: Princeton University Press

Damuth, J., \& Heisler, I. L. (1988). Alternative formulations of multilevel selection. Biology and Philosophy, 3(4), 407-430. doi: $\underline{10.1007 / \mathrm{BF} 00647962}$ 
Dugatkin, L. A., \& Reeve, H. K. (1994). Behavioral ecology and levels of selection: dissolving the group selection controversy. Advances in the Study of Behavior, 23, 101-133. doi: 10.1016/S0065-3454(08) $\underline{60352-6}$

Fisher, R. A. (1970). The design of experiments (8th ed.). New York: Hafner.

Gardner, A., \& Foster, K. R. (2008). The evolution and ecology of cooperation-History and concepts. In J. Korb \& J. Heinze (Eds.), Ecology of social evolution (pp. 1-36). Heidelberg: Springer. doi: $\underline{10.1007 / 978-3-540-75957-7 \quad 1}$

Godfrey-Smith, P. (2009). Darwinian populations and natural selection. New York: Oxford University Press, USA.

Godfrey-Smith, P. (2011). Darwinian populations and transitions in individuality. In B. Calcott \& K. Sterelny (Eds.), The Major Transitions in Evolution Revisited. Cambridge, MA: MIT Press. doi: 10.7551/ mitpress/9780262015240.003.0005

Grantham, T. A. (1995). Hierarchical Approaches to Macroevolution: Recent Work on Species Selection and the "Effect Hypothesis". Annual Review of Ecology and Systematics, 26, 301-321. doi: 10.1146/ annurev.es.26.110195.001505

Griesemer, J. R. (2000). The units of evolutionary transition. Selection, 1(1), 67-80. doi: 10.1556/Select. $1.2000 .1-3.7$

Griesemer, J. R. (2014). Reproduction and the Scaffolded Development of Hybrids. In L. R. Caporael, J. R. Griesemer \& W. C. Wimsatt (Eds.), Developing Scaffolds in Evolution, Culture, and Cognition (pp. 23). Cambridge, MA: MIT Press.

Hamilton, W. D. (1964). The genetical evolution of social behaviour. I. Journal of theoretical Biology, 7(1), 1-16. doi: 10.1016/0022-5193(64)90038-4

Hull, D. L. (1975). Central subjects and historical narratives. History and Theory, 14(3), 253-274. doi: $\underline{10.2307 / 2504863}$

Jablonski, D. (2008). Species selection: theory and data. Annual Review of Ecology, Evolution, and Systematics, 39, 501-524. doi: 10.1146/annurev.ecolsys.39.110707.173510

Kerr, B., \& Godfrey-Smith, P. (2002). Individualist and multi-level perspectives on selection in structured populations. Biology and Philosophy, 17(4), 477-517. doi: 10.1023/A:1020504900646

Krimbas, C. B. (2004). On fitness. Biology and Philosophy, 19(2), 185-203. doi: 10.1023/B:BIPH. 0000024402.80835.a7

Lloyd, E. A. (1988). The structure and confirmation of evolutionary theory. New York: Greenwood Press.

Lloyd, E. A., \& Gould, S. J. (1993). Species selection on variability. Proceedings of the National Academy of Sciences, 90(2), 595-599. doi: 10.1073/pnas.90.2.595

Lloyd, E. A., Lewontin, R. C., \& Feldman, M. W. (2008). The Generational Cycle of State Spaces and Adequate Genetical Representation. Philosophy of science, 75(2), 140-156. doi: 10.1086/590196

Maynard Smith, J. (1978). The evolution of sex. Cambridge: Cambridge University Press.

Michod, R. E. (1999). Darwinian dynamics. Princeton: Princeton University Press.

Michod, R. E. (2005). On the transfer of fitness from the cell to the multicellular organism. Biology and Philosophy, 20(5), 967-987. doi: 10.1007/s10539-005-9018-2

Michod, R. E. (2011). Sex and multicellularity as evolutionary transitions in individuality. In B. Calcott \& K. Sterelny (Eds.), The Major Transitions in Evolution Revisited. Cambridge, MA: MIT press. doi: $10.7551 /$ mitpress $/ 9780262015240.003 .0010$

Michod, R. E., Nedelcu, A. M., \& Roze, D. (2003). Cooperation and conflict in the evolution of individuality: IV. Conflict mediation and evolvability in Volvox carteri. BioSystems, 69(2-3), 95-114. doi: 10.1016/S0303-2647(02)00133-8

Michod, R. E., \& Roze, D. (1999). Cooperation and conflict in the evolution of individuality. III. Transitions in the unit of fitness. In C. L. Nehaniv (Ed.), Mathematical and Computational Biology: Computational Morphogenesis, Hierarchical Complexity, and Digital Evolution (pp. 47-92). Providence, Rhode Island: American Mathematical Society.

Michod, R. E., Viossat, Y., Solari, C. A., Hurand, M., \& Nedelcu, A. M. (2006). Life-history evolution and the origin of multicellularity. Journal of theoretical Biology, 239(2), 257-272. doi: $10.1016 / \mathrm{j} . j \mathrm{jtbi}$. $\underline{2005.08 .043}$ 
Okasha, S. (2006). Evolution and the Levels of Selection. New York: Oxford University Press, USA. doi: 10.1093/acprof:oso/9780199267972.001.0001

Okasha, S. (2009). Individuals, groups, fitness and utility: multi-level selection meets social choice theory. Biology and Philosophy, 24(5), 561-584. doi: 10.1007/s10539-009-9154-1

Okasha, S. (2011). Reply to Sober and Waters. Philosophy and Phenomenological Research, 82(1), 241-248. doi: $10.1111 / \mathrm{j} .1933-1592.2010 .00474 . \mathrm{x}$

Pearl, J. (2000). Causality: models, reasoning and inference. Cambridge: Cambridge University Press.

Pocheville, A. (2010). What Niche Construction is (not). La niche écologique: concepts, modèles, applications. PhD thesis. Ecole Normale Supérieure. Paris.

Shipley, B. (2002). Cause and correlation in biology: a user's guide to path analysis, structural equations and causal inference. Cambridge: Cambridge University Press.

Sober, E. (2001). The two faces of fitness. In R. S. Singh, C. B. Krimbas, D. B. Paul \& J. Beatty (Eds.), Thinking about evolution: historical, philosophical, and political perspectives. Cambridge: Cambridge University Press.

Sterelny, K. (1996). The return of the group. Philosophy of Science, 63(4), 562-584. doi: 10.1086/289977

Stidd, B. M., \& Wade, D. L. (1995). Is species selection dependent upon emergent characters? Biology and Philosophy, 10(1), 55-76. doi: 10.1007/BF00851987

Thoday, J. (1953). Components of fitness. Symposium of the Society for Experimental Biology, 7, 96-1 13.

Van Valen, L. M. (1989). Three paradigms of evolution. Evolutionary theory, 9(1), 1-17.

Vrba, E. S. (1983). Macroevolutionary trends: new perspectives on the roles of adaptation and incidental effect. Science, 221(4608), 387-389. doi: 10.1126/science.221.4608.387

Vrba, E. S. (1989). Levels of selection and sorting with special reference to the species level. Oxford Surveys of Evolutionary Biology, 6, 111-168.

Waters, K. C. (2011). Okasha's Unintended Argument for Toolbox Theorizing. Philosophy and Phenomenological Research, 82(1), 232-240. doi: 10.1111/j.1933-1592.2010.00472.x

Weismann, A. (1893). The germ-plasm: a theory of heredity. New York: C. Scribner's sons. doi: 10.5962/ bhl.title.88042

West, S. A., Griffin, A. S., \& Gardner, A. (2007). Social semantics: altruism, cooperation, mutualism, strong reciprocity and group selection. Journal of Evolutionary Biology, 20(2), 415-432. doi: 10.1111/j. 1420-9101.2006.01258.x

West, S. A., Lively, G. M., \& Read, A. F. (1999). A pluralist approach to sex and recombination. Journal of Evolutionary Biology, 12(6), 1003-1012. doi: 10.1046/j.1420-9101.1999.00119.x

Woodward, J. (2003). Making things happen: A theory of causal explanation. New York: Oxford University Press, USA.

Woodward, J. (2013). Causation and manipulability. Stanford encyclopedia of philosophy. Retrieved from http://plato.stanford.edu/archives/win2013/entries/causation-mani/

\section{Notes}

1. The distinction between particle and collective comes from Okasha $(2006,4)$.

2. In the rest of the paper I at times claim that MLS1 and MLS2 are commensurable. This should only be understood in the context of Okasha (2006) and Michod (2005) where they propose to give an ontology to MLS1 and MLS2. It should be clear that Damuth and Heisler's initial distinction only regards MLS1 and MLS2 as two possible methods to study multilevel selection. They write: "Once one has decided to analyze a given situation in terms of multilevel selection processes both approaches [MLS1 or MSL2] are legitimate within that context and a choice has to be made depending upon what questions are of interest" (Damuth and Heisler 1988, 411).

3. A cross-level by-product is defined by Okasha $(2006,76)$ as "a side effect, or by-product, of selection acting at a different level".

4. Michod and colleagues use the word 'decoupling' to refer to this phenomenon. By decoupling they mean that the fitness at the collective level becomes expressed in a different currency than fitness at the particle level and is not translatable into fitness at that level.

5. Okasha and Michod use "fecundity" instead of "fertility". These terms are equivalent.

6. There is, however, no scope for generality in Michod's models. 
7. See for instance the cases of prions and LINE transposon provided by Godfrey-Smith (2009, 80). Kin selection, which is standard evolutionary theory, represents another example in which the reproduction of one allele is done "indirectly"; that is, without material overlap (e.g., sterile workers helping the queen). Kin selection is a widely accepted model in evolutionary theory and thus represents, for my purpose, the clearest case of the sort of formal reproduction necessary and sufficient for evolution by natural selection to occur.

8. This is to be taken here as a metaphor.

9. Incidentally, the model proposed here is incompatible with Weismann's germ-plasm theory (1893), which is widely accepted in some subdisciplines of evolutionary biology. Yet in the context of evolutionary transitions of individuality, Buss $(1983,1987)$ has conclusively shown that early germ soma separation was only one minority mode of development once all the taxa on Earth are taken into account.

10. For a survey of the issues surrounding the evolution of sex see West, Lively, \& Read (1999) and the different commentaries on their article.

11. Some Authors (e.g., Damuth \& Heisler 1988; Lloyd 1988; Lloyd \& Gould 1993) have questioned the validity of Vrba's acid test and more generally what she calls "effect macroevolution" (Vrba 1983). However, Okasha (2006, 207), relying on Grantham's (1995) and Stidd and Wade's (1995) analyses, shows that Vrba's test is valid. The only problem with the test according to Okasha, is that it should not focus on emergent properties but emergent level of selection. Although it is not clear how emergent levels of selection could be independent from the properties of fitness bearers, for the sake of the argument I accept that Vrba's test is valid and focus here on the notion of emergent level of selection.

12. Pearl (2000) and Shipley (2002) provide sophisticated statistical tools that rely, among other things, on the simple idea that correlation does not equate to causation for situations in which experimentally controlled and randomized experiments are impossible.

13. This will of course be a problematic assumption in many cases, but considering a multicellular organism to be made of cells and part of the cellular environment should be seen as the result of a change in grain of descriptions, not a change in level of selection.

14. Since 6 cells are produced over 5 cell generations this means that on average a cell produces 1.43 offspring cells per cell generation.

\section{AGKNOWLEDGMENTS}

I am thankful to Paul Griffiths, Adam Hochman,and Arnaud Pocheville for their comments on an earlier version of this paper. I am also grateful to Johann Hariman and Robyn Kath who proofread the English of the paper. This research was supported under Australian Research Council's Discovery Projects funding scheme (project number DP0878650) and an International Postgraduate Research Scholarship from the University of Sydney.

Copyright (C) 2015 Author(s).

This is an open-access article distributed under the terms of the Creative Commons Attribution-NonCommercial-NoDerivs license, which permits anyone to download, copy, distribute, or display the full text without asking for permission, provided that the creator(s) are given full credit, no derivative works are created, and the work is not used for commercial purposes.

ISSN 1949-0739 